\title{
Technical and Tactical Command Decision Algorithm of Football Matches Based on Big Data and Neural Network
}

\author{
Lei Fang, ${ }^{1}$ Qiang Wei, ${ }^{2}$ and Cheng Jian $\mathrm{Xu}$ (D) $^{3}$ \\ ${ }^{1}$ Northwest University for Nationalities, Lanzhou 730124, Gansu, China \\ ${ }^{2}$ Department of Physical Education, Tangshan Normal University, Tangshan, China \\ ${ }^{3}$ Department of Competitive Sports, Guangdong Sports Vocational and Technical College, Guangzhou 510663, Guangdong, China
}

Correspondence should be addressed to Cheng Jian Xu; haohaoxuexi86668@163.com

Received 21 January 2021; Revised 2 March 2021; Accepted 20 March 2021; Published 8 April 2021

Academic Editor: Shah Nazir

Copyright ( $) 2021$ Lei Fang et al. This is an open access article distributed under the Creative Commons Attribution License, which permits unrestricted use, distribution, and reproduction in any medium, provided the original work is properly cited.

\begin{abstract}
A successful football team not only consists of more than a dozen people on the field but also includes a complete training, analysis, coaching team behind it, and the same basic education and youth training system. With the development of scientific concepts and the advancement of computer technology, more people have begun to study the use of modern technology to replace part of the traditional human work with low creativity and the use of more convenient quantitative analysis, prediction and other technologies to assist football professionals' decision-making. Based on big data and neural network technology, this paper has designed a novel football technical and tactical command decision algorithm. First, the use of big data technology for analyzing the characteristics of the historical big data of football competitions provides valuable data for the work of this article. Secondly, to formulate scientific and reasonable football technical and tactical command, it requires learning effective offensive or defensive strategies from the big data of football competitions. This article uses deep neural networks to learn massive amounts of football competition data, which can effectively predict the offensive and defensive tactics of each position of the team to a certain extent. In addition, in order to better learn the timing video data of football matches, this paper also has proposed to use long- and shortterm memory networks to improve the algorithm of this paper. The proposed method has achieved good results in football technical and tactical command and decision-making and also provides some new ideas for the subject of football combined with computer technology.
\end{abstract}

\section{Introduction}

Football match is a popular sport activity around the world. The prosperous development of sports business and the application of Internet technology allow fans to easily obtain high-quality videos of almost all professional football matches. Data providers can easily collect and archive these video data as well as game and player statistics. However, the resulting big data has brought both challenges and opportunities for sports professionals and related companies. In order to make the most of these data, football scouts and coaches need advanced analytical tools to evaluate, select, and train players. Researchers have been developing tools for scientific analysis to help evaluate the performance of players and teams [1].
Although the relevant research is quite popular and has made certain achievements, its scientificity and effectiveness still need to be improved [2-6]. Existing research is usually difficult for professionals and amateurs to examine. It is not easy to conduct professional and reliable quantitative analysis of competition data. The football game is a complex whole, affected by many factors. On the other hand, statistics are sparse and lack relevance. It is not wise to use only shooting or passing statistics to describe a game. Most existing systems only use statistics instead of considering the whole, which may lead to a misunderstanding of the game. This is why many coaches still believe in their eyes rather than statistics. Football professionals and computer and data researchers often lack effective communication, which makes the development of related tools inseparable from the 
long-term close cooperation between football professionals and computer and data researchers. Finally, big data and neural network [7-12] technologies still need indepth research in the football field. As a football game with many players on the field, rich tactics, and a large range of activities, its flexibility and complexity make many existing methods unable to directly hit the core of football. Researchers need to develop relevant neural network algorithms specifically for football sports, taking full account of the particularity of football sports and filling the gaps in the industry.

Therefore, this paper designs a novel football technical and tactical command decision algorithm based on big data and neural network technology [13-17]. First of all, the use of big data technology to analyze the characteristics of the historical big data of football competitions provides a lot of valuable data for the work of this article. Secondly, to formulate scientific and reasonable football technical and tactical command, it requires learning effective offensive or defensive strategies from the big data of football competitions. This article uses deep neural networks to learn massive amounts of football competition data, which can effectively predict the offensive and defensive tactics of each position of the team to a certain extent. In addition, in order to better learn the timing video data of football matches, this paper also proposes to use long- and short-term memory networks to improve the algorithm of this paper. Following are the main innovative points of this paper:

(1) This paper uses big data technology to analyze the characteristics of massive historical data of football matches, extracts a lot of valuable data, and designs some new interactive methods, quantitative methods, and evaluation methods for football matches

(2) We have innovatively introduced a long- and shortterm memory network (LSTM) to learn the time series data of football matches and built a deep neural network model to assist football technical and tactical command decisions

(3) We have conducted sufficient comparative experiments and ablation studies to prove the effectiveness of the football technique and tactics command decision algorithm based on big data and neural network proposed in this paper, which can provide a scientific basis for sports coaches to formulate reasonable football techniques and tactics

The organization of the paper is as follows: Section 2 shows the related work to the proposed study. Section 3 represents the methodology section of the paper. Section 4 depicts the experiment and results of the paper. The paper is concluded in Section 5.

\section{Related Work}

Various approaches and techniques are existing in literature associated with diverse decisions of football matches. Krzysztof and Pawet [18] conducted a multidimensional study on the characteristics of goals in the game. The author believes that insufficient sample size will make the research results inaccurate. In the 08/09 season, the Premier League's close-range shots accounted for more than $50 \%, 53 \%$ of the goals were below the goalkeeper's stock, and the second half of the goal accounted for $57 \%$. The author believes that the inattention of players due to fatigue is a goal loss. Key factor: the goal rate after dribbling was only $12.8 \%$. The goalkeeper failed to save a low shot $(53 \%$ of goals). In general, the author believes that the lower area of the upper goal is the most effective area for scoring. Michalls et al. [19] through the way of creating goals in the Premier League, La Liga, Bundesliga, and Serie A, the purpose of the study is to compare the creation of goals in the four major European football leagues. The sample includes 80 random matches in the 2017-2018 season (20 La Liga; 20 Premier League; 20 Bundesliga; 20 Italian Serie A). 914 teams created scoring opportunities in the game. The study uses a multidimensional observation method to evaluate multiple tactical dimensions and the three stages of the team's beginning, development, and end. Kruskal-Wallis analysis showed that there were significant tactical differences in these 4 games. La Liga shows a greater proportion of long-distance offenses and teamwork. The Premier League appears to be quick and direct in attacking methods. Compared with cooperating offense and quick offense, Bundesliga has the most counterattacks, Serie A has the shortest offensive distance, and the proportion of counterattacks and direct attacks is also larger. Kubayi [20] studied the goal pattern of the 2018 World Cup. All goals in the game are analyzed with the InStat video analysis system. The results showed that a total of 169 goals were scored in this game (sports battle: 60.9\%; setup: $39.1 \%)$. Eighty-five goals $(82.5 \%)$ came from the team's offense, and 18 goals (17.5\%) came from the counterattack. Chi-square test shows that there is a significant difference in the type of possession (card $2(1, n=103)=43.58$, $p \leq 0.001)$. Compared with the first third (33\%) and the middle third (32\%), the last third (35\%) has the most goals. The results also showed that most goals came from short passes $(69.9 \%), 13.6 \%$ came from long passes, and $16.5 \%$ came from mixed passes (chi $2(2, n=103)=62.12$, $p \leq 0.001)$.

Perl et al. [21] believes that "different technologies have different data collection methods. The technical collection methods can be divided into two types of technologies: video capture and wearable transmission." Video capture uses panoramic photography and game video recording. The latter places chips in clothes and sneakers to analyze sports performance. Reilly and Williams [22] found that the Cologne University of Sport used a computer system to process video matches and found that, before $80 \%$ of a goal, the number of passes is no more than 3 times. Fast attack and teamwork have always been the direction of modern football development. Pollard and Reep [23] found that the analysis of football performance is divided into two types: predata collection and postanalysis. Compared with physical load, technical and tactical analysis can determine the outcome of a match. 


\section{Methodology}

Football technique is the general term for reasonable actions and movements used in football matches. According to the analysis characteristics of large-scale competitions in the physical education field and the realistic possibilities of video statistics, this research divides football skills into two categories: offensive technology and defensive technology. Offensive skills include passing, ball possession, dribbling breakthrough, and shooting. Defensive skills include steals, clearances, and tackles. Among these concepts, the concepts of passing, possession, and shooting are very clear. Figures 1(a)-1(d) show an example of dribble breakthrough attack.

The algorithm proposed in this paper is shown in Figure 2. We define the other concepts in detail as follows:

(1) Dribble breakthrough: the player uses dribbling or passing to pass one or more opponents' pressing and blocking forward

(2) Steal: when the attacking player holds the ball or the attacking player passes the ball to his partner, the defensive player uses defensive skills to get the ball before the opposing player

(3) Rescue: in order to prevent the attacking side from attacking, the defensive player directly destroys the ball out of the defensive area without passing the ball.

3.1. Stadium Modeling. The purpose of stadium modeling is to convert the position of the player from the video perspective to the position of the top view (aerial view). One of the purposes of this is that redundant video information is often not needed in football visualization analysis, but top view and representative players are used. The dynamic icon is replaced. The second reason is that, in order to obtain information about the physical fitness of the player in the future, the player's position information needs to be obtained first. Due to the reason that the distance is small, the position information obtained by simply tracking the original video cannot be used for calculation. Therefore, perspective transformation is required for football field modeling. In this study, both the video provided by the Shanghai Institute of Physical Education and the video of the National Games adopted the FIFA standard show that the vertical length of the field is 105 meters, the horizontal width is 68 meters; the goal: the length is 7.32 meters, the height is 2.44 meters; the big penalty area (penalty area): horizontal 40.32 meters, vertical 16.5 meters, on the bottom line 5 meters away from the goal post; small restricted area (goal area): 18.32 meters long, 5.5 meters wide, 5.5 meters from the goal post on the bottom line; center circle area: a radius of 9.15 meters; 4 corner kick area: radius of 1 meter, 13.84 meters from the penalty area; penalty kick arc: a semicircle with a radius of 9.15 meters centered on the penalty kick point; penalty kick point: 11 meters from the goal line. For the convenience of debugging in the development process and the applicability of the subsequent software release, our video size and court plane model diagram are both $1920 * 1080$ pixels in size.
After the target detection and tracking part of the video, we successfully obtained the pixel position $(x, y)$ of each player. Before we can use this location, there is one more step that needs further research. When the human eye sees things nearby, they appear larger than those far away. This is often referred to as a perspective phenomenon. The perspective transformation refers to the transfer of an object from one state to another. Popular application examples such as in the picture, there is a trapezoidal test paper viewed from an oblique direction. Its actual shape is undoubtedly a rectangle. Through perspective transformation, we select several points on the test paper and then select the corresponding one on the plan view. To demonstrate, the rectangular test paper in the illustration can be turned into a rectangle when viewed from the front. At this time, if you want to recognize the words on the test paper, the effect will be better. At the same time, the points on the plane where the test paper is not are used because of the number. The correct perspective transformation produces distortion. In general, the perspective conversion process converts a $3 \mathrm{D}$ world to a $2 \mathrm{D}$ image, but we need to determine a plane that is the only concern. Points outside the plane will be distorted. In this article, we need to use such a transformation matrix to adjust the pixel position as follows. Through perspective transformation, we obtain a top view. In the top view, any two points with the same distance in the real world have the same Euclidean distance between their pixels. We can superimpose the distance between two points between adjacent frames to calculate the running distance of the player. The essence of perspective transformation is to change the projection of the image to a new perspective plane. The general conversion formula can be written as, where $(u, v)$ are the pixel coordinates of the original image, $\left(x=\left(x^{\prime} / w^{\prime}\right), y=\left(y^{\prime} / w^{\prime}\right)\right)$. It is the pixel coordinate transformation after the image. The perspective transformation matrix is shown as follows:

$$
\left[x^{\prime}, y^{\prime}, w^{\prime}\right]=[u, v, w]\left[\begin{array}{lll}
p_{11} & p_{12} & p_{13} \\
p_{21} & p_{22} & p_{23} \\
p_{31} & p_{32} & p_{33}
\end{array}\right] \text {. }
$$

We choose four pixels in the original image, which are usually easily distinguishable signs such as the corner, intersection, or inflection point of the court. Then, we find their corresponding points in the model image. We use these two sets of points to calculate the transformation matrix. After the transformation matrix is obtained, we select the position of the player's foot that is on the same plane as the court for transformation to obtain the position of the top view.

In the game, football can easily be in the air. If we use the same method as calculating the player's position, the height of the football will cause a big error: the position obtained by the perspective transformation is the position of the football projected on the ground plane in the video. How do we find the exact position of football? First of all, we need to calibrate the camera parameters of the camera used to obtain the correspondence between camera coordinates and world coordinates. After having at least two different views and calibrating the camera, we can calculate the $3 \mathrm{D}$ position of 


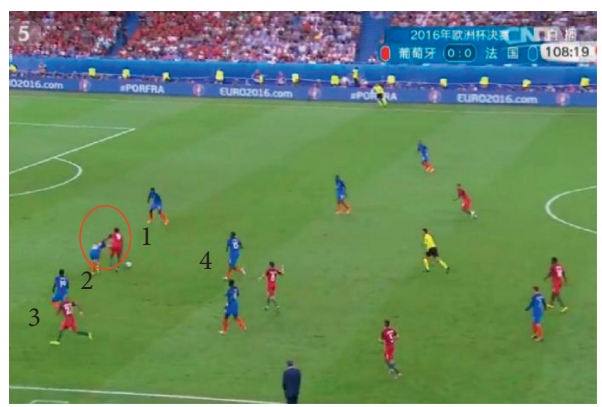

(a)

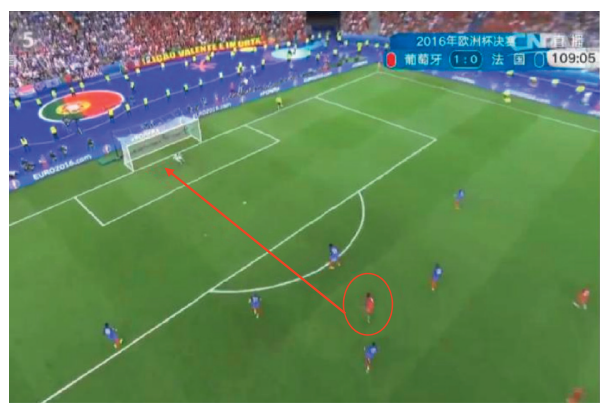

(c)

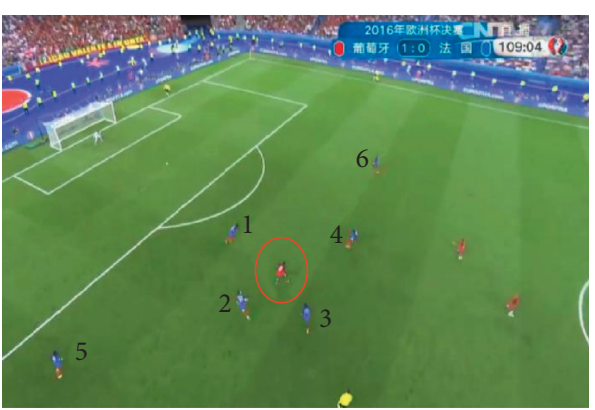

(b)

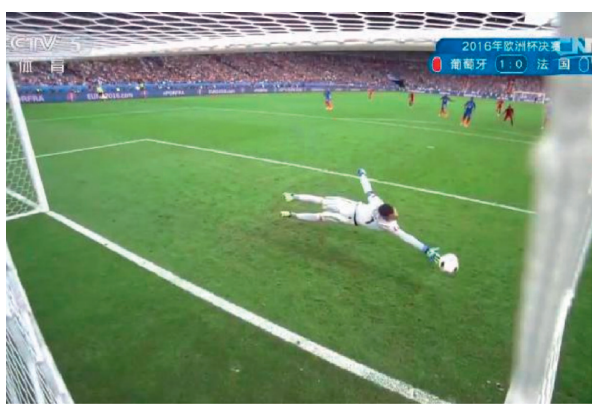

(d)

Figure 1: Example of dribble breakthrough attack. (a) Striker takes the ball. (b) Striker gets rid of. (c) Forward shot. (d) Goal.

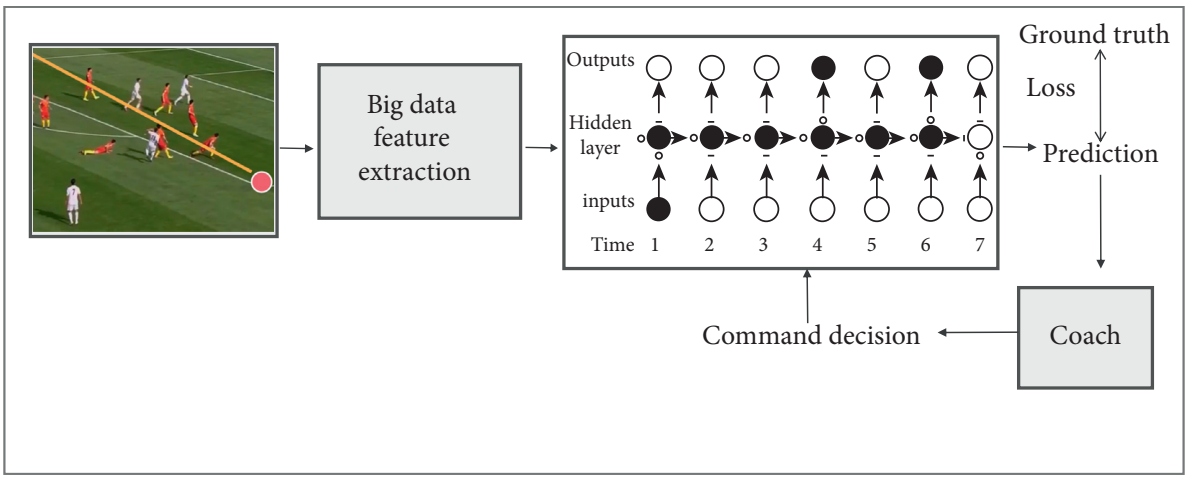

FIGURE 2: The flowchart of the overall architecture of our algorithm.

the ball. This is a simple application of $3 \mathrm{D}$ reconstruction. Suppose the straight line $L$ can be expressed by the following formula:

$$
L=t+w_{3}^{*} d_{3}
$$

where $t$ is the position of the camera, $w_{3}$ is a constant, and direction 3 represents the direction of the line. We have two cameras, which form two nonparallel lines in the coordinate system:

$$
\left\{\begin{array}{l}
L_{1}=t_{1}+w_{1}^{*} d_{1} \\
L_{2}=t_{2}+w_{2}^{*} d_{2}
\end{array}\right.
$$

Calculate the intersection point:

$$
\left\{\begin{array}{l}
d^{*} d_{3}=0, \\
d_{2}{ }^{*} d_{3}=0
\end{array}\right.
$$

After the calibration operation is completed, with the help of perspective transformation, we can have two lines, which can be understood as a light taken by the football by the camera. Due to the influence of the height of the football mentioned above, the actual position of the football is on this line. At some point, ideally, the 3D position $(x, y, z)$ of a football can be calculated from the intersection of two lines. In practical applications, it is impossible because the two lines intersect. We use the midpoint of the shortest distance line of the two straight lines to simulate the intersection of the two straight lines to obtain the football position.

3.2. Recurrent Neural Network. Deep neural networks [24] refer to neural networks with deep network structure, which have made outstanding achievements in match prediction [25-29] and computer vision [30-34]. Deep neural networks have more neural network layers, so they can extract more 
features from the input and have a stronger ability to portray reality. According to the core network used, deep neural networks can be divided into deep convolutional neural networks (deep CNN) and deep recurrent neural networks (deep RNN). In the field of computer vision and image and video processing, convolutional neural networks have greater advantages than other neural networks. In the field of sports competition data analysis, because the input data is dependent and sequential mode, there is no correlation between the front and back inputs of CNN, and all outputs are independent of each other, so the performance of $\mathrm{CNN}$ is not good. For the task of automatic generation of Chinese couplets studied in this article, all outputs are related to the previous output, and some biases based on the previous output information are required. Therefore, the recurrent neural network RNN is more suitable.

3.2.1. Standard Recurrent Neural Network. The basic neural network includes a three-layer structure of input layer, hidden layer, and output layer. It only establishes connections between layers, while the standard recurrent neural network RNN (recurrent neural network) is based on this, in the same layer. Connections are also established between neurons. The neural network structure of the RNN is shown in Figure 3. The right side of the equal sign is the timeexpanded diagram of the neural network, and the left side of the equal sign is its simplified diagram. Assuming that $x_{t}$ is the input of time step $t$ in the sequence and $h_{t}$ is the hidden state vector of that time step. According to the neural network structure in Figure 3, the current $h_{t}$ is

$$
h_{t}=F\left(x_{t} W_{x h}+h_{t-1} W_{h h}+b_{h}\right) \text {, }
$$

where $h_{t-1}$ is the hidden state vector of the previous time step. In simple terms, the hidden state vector of time step $t$ is determined by the input $x_{t}$ of the current time step and the hidden state vector $h_{t-1}$ of the previous time step. $F$ is the activation function of the neural network, and $w_{x h}$ and $w_{h h}$ are the weight matrices of the neural network.

3.2.2. Long- and Short-Term Memory Network. The longand short-term memory network (As shown in Figure 4) is more suitable for solving the time series problem with longterm dependence, which is to solve the shortcomings of the general RNN network. As shown in Figure 5, compared with ordinary RNN, LSTM adds a memory unit and three controllers: input control, forgetting control, and output control. The function of the memory unit is to store the network state; the input controller decides how much input is kept at the current moment; the forgetting controller decides the degree of retention of the neural network state at the current moment; the output controller determines the output information according to the neural network state at the current moment.

The core of LSTM is the memory cell state, which is composed of a sigmoid network and a multiplier. Using this structure, the information in the memory cell can be added or deleted. The output range of the sigmoid network is $[0,1]$, which indicates how much information can pass through the memory unit, 0 means none can pass, and 1 means all can pass.

Forgetting gate: use a sigmoid layer to receive the output of the previous time node $t-1$ and the input of the current time node $t$, merge it into a tensor, and then apply a linear transformation afterwards. After the sigmoid activation function, the output of the forgetting gate is a value between 0 and 1 . This value will be multiplied by the internal state, which is why it is called the forget gate. If $f_{t}=0$, the previous internal state is completely forgotten, and if $f_{t}=1$, it will pass without any change. The calculation equation of the forget gate is as follows:

$$
f_{t}=\sigma\left(W_{f}\left[h_{t-1}, x_{t}\right]+b_{f}\right) .
$$

Input gate: accept previous output and new input and pass it to another sigmoid layer. The input gate returns a value between 0 and 1 . Then, the value returned by the input gate is multiplied with the output of the candidate layer.

$$
i_{t}=\sigma\left(W_{i}\left[h_{t-1}, x_{t}\right]+b_{i}\right)
$$

This layer mixes the input with the output of the previous layer, then applies hyperbolic tangent activation, and returns a candidate vector to add to the internal state. The internal status update rules are as follows:

$$
\begin{array}{r}
C_{t}=\tanh \left(W_{C}\left[h_{t-1}, x_{t}\right]+b_{C}\right), \\
i_{t}=f_{t}{ }^{*} C_{t-1}+i_{t}{ }^{*} C_{t} .
\end{array}
$$

The output gate controls how many internal states are passed to the output, and it works like other gate structures. The output gate calculation equation is as follows:

$$
\begin{array}{r}
O_{t}=\sigma\left(W_{o}\left[h_{t-1}, x_{t}\right]+b_{o}\right), \\
h_{t}=O_{t}{ }^{*} \tanh C_{t} .
\end{array}
$$

The above is the structure principle of LSTM.

\section{Experiments and Results}

The following sections show the experiments and results of the paper.

4.1. Experimental Environment. Since the experiment in this article needs to train a deep neural network, the scale is large, the structure is more complex, and the calculation scale is large. The neural network training process needs to use GPU to accelerate the calculation. The experimental environment configuration is shown in Table 1.

The experiments in this article are all done on this machine, where the neural network training is accelerated by GPU, the programming language used is Python, the version is 3.6, the deep learning framework used is Keras2.1.5, and the IDE for program deployment is PyCharm.

4.2. Hyperparameter Settings. Use LSTM as the neural network structure of the encoder and decoder and share parameters between the SoftMax layer and the word vector 


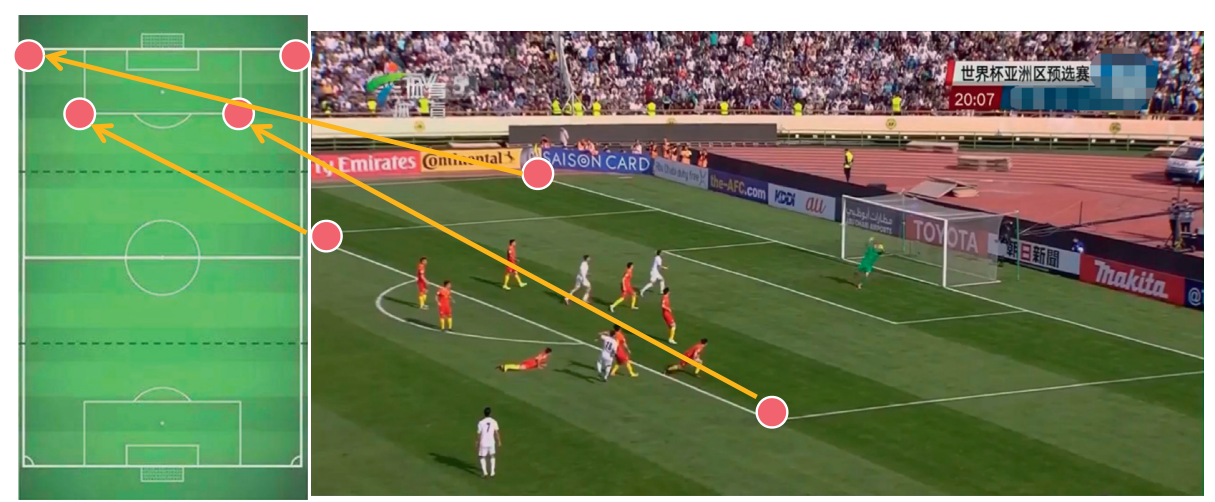

Figure 3: Goal retrieval.

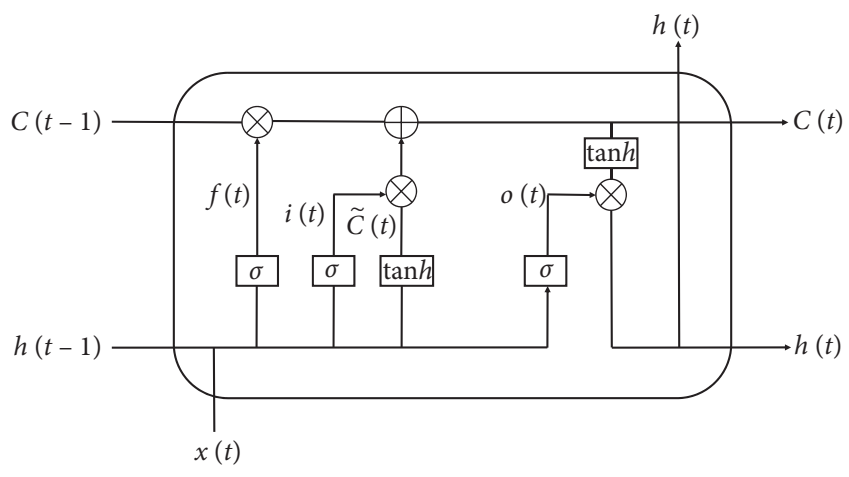

Figure 4: Basic structure of long- and short-term memory network (LSTM).

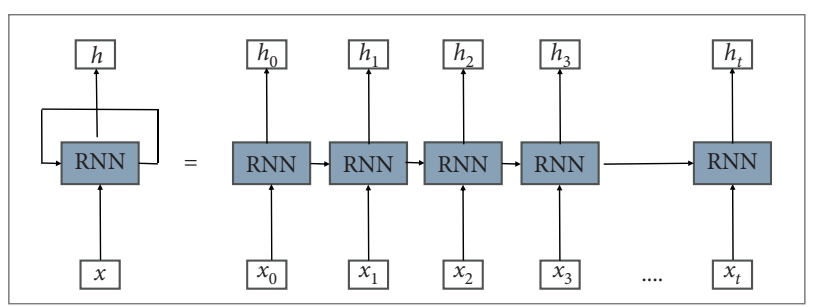

FIGURE 5: Basic structure of standard recurrent neural network.

layer to reduce the number of parameters [[45][46]\}. The size of the LSTM hidden layer is 1024, the encoding and decoding neural network, the number of layers in the LSTM structure is 6 layers, the batch size is 128 , the probability of a node being dropped out is set to 0.1 , and the model is saved every 2000 steps in training.

4.3. Experimental Results of Different Methods. In this section, experimental results are presented to verify the proposed system. This paper presents experimental results to verify the proposed system. In order to ensure the effectiveness of the method, the detection experiment was applied to the football video data of the 13th China National Games. In order to prove the effectiveness of the width learning system, we compare our method with existing mainstream methods, including SVM, SRC, LRC, LCCR, and RDBLS.
TABLE 1: Experimental environment configuration.

\begin{tabular}{lc}
\hline Operating system & Windows \\
\hline CPU & Intel (R) Core (TM)i7-8700 CPU @ \\
GPU & $3.20 \mathrm{GHz}$ \\
RAM & NVIDIA GeForce GTX 1080 \\
Deep learning & $16 \mathrm{~GB}$ \\
library & Keras2.1.5 \\
\hline
\end{tabular}

Table 2 gives a quantitative comparison of different methods. In Table 2, we find that our method can achieve less training time, while the accuracy remains at a level similar to other methods. The results of the number of different mapped features are given in Table 3. In Table 2, we tested three features: low-frequency Fourier transform feature (FFT), Gabor amplitude, and local binary pattern (LBP) instead of using the original pattern unaligned image. These methods are all suitable for identification with the information of the previous sequence. We tested the success rate of the tracker when it lost the target. In short, the width learning method showed acceptable accuracy and excellent running time.

Passing is one of the most basic techniques in football. Players need to pass the ball to connect in series. Passing is also the basis of collective cooperation in football. To complete tactical coordination and even create shooting opportunities, a team needs to pass the ball. Since the European Cup in 2008, the World Cup in 2010, and the European Cup in 2012, after the Spanish team won successively, a passing style has been set off in the world football. The world's strong teams have paid more and more attention to the passing ability of the players. Teams generally have a pass success rate of more than $90 \%$.

The statistics of the number of passes, the number of long passes and the success rate of passes of the Portuguese team in each game of this European Cup are made. The specific results are shown in Table 2. The overall pass success rate of the Portuguese team is not high, with an average success rate of only $80.86 \%$. In the last game of the group match, the Portuguese team had a traditional success rate of only $64 \%$ against the weak Hungary team. The passing success rate is not high but the game can be won, which has become a common phenomenon in recent world high-level football 
TABLE 2: Statistics of passing characteristics.

\begin{tabular}{lccc}
\hline Competitor & Total number of passes & Long passes & Pass success rate (\%) \\
\hline Iceland & 421 & 62 & 88.8 \\
Austria & 403 & 53 & 83.9 \\
Hungary & 437 & 67 & 64.0 \\
Croatia & 489 & 100 & 80.9 \\
Poland & 561 & 83 & 82.3 \\
Wales & 587 & 58 & 82.7 \\
France & 506 & 98 & 83.4 \\
\hline
\end{tabular}

TABle 3: Predict the success rate of retrieval.

\begin{tabular}{|c|c|c|c|c|c|c|c|}
\hline \multirow{2}{*}{ Methods } & \multicolumn{2}{|c|}{ FFT } & \multicolumn{2}{|c|}{ Gabor } & \multicolumn{2}{|c|}{ LBP } & \multirow{2}{*}{ Mean } \\
\hline & Probability (\%) & Running time & Probability (\%) & Running time & Probability (\%) & Running time & \\
\hline SVM & 15.8 & 22.6 & 49.8 & 16.4 & 27.7 & 20.36 & 31.1 \\
\hline SRC & 37.4 & 3682.9 & 65.7 & 3587.2 & 58.6 & 3608.2 & 53.9 \\
\hline LRC & 20.3 & 31.72 & 26.4 & 30.02 & 28.4 & 33.79 & 25.0 \\
\hline LCCR & 27.8 & 20.09 & 58.6 & 21.65 & 64.2 & 21.23 & 50.2 \\
\hline RDBLS & 45.7 & 0.58 & 70.2 & 0.73 & 69.3 & 0.67 & 61.7 \\
\hline Ours & 46.9 & 0.55 & 72.2 & 0.68 & 70.1 & 0.66 & 62.9 \\
\hline
\end{tabular}

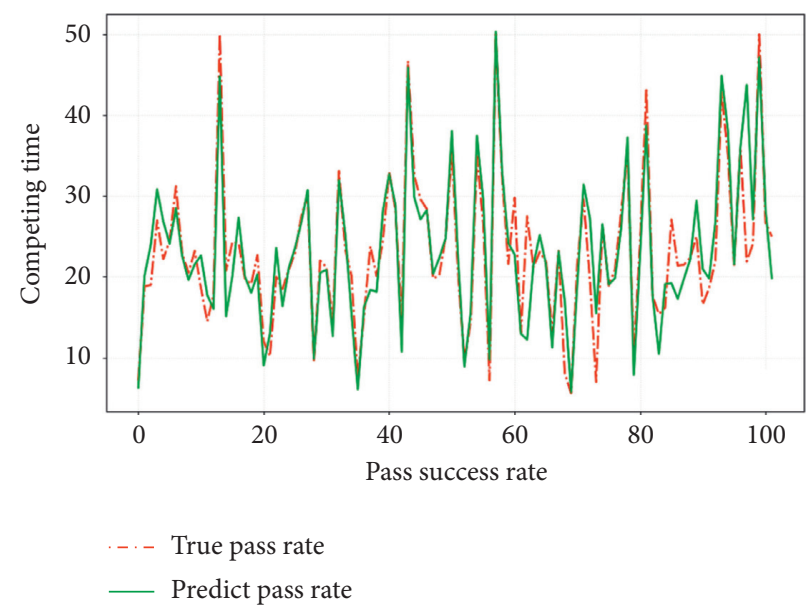

FIgURE 6: The comparison result of the Portuguese team's passing rate prediction using our algorithm.

matches. The reason is that the teams are not blindly pursuing a safer backcourt in the offense. It is daring to pass the threatening ball in the offense. In this case, although the turnover rate is high, the threat is not reduced at all.

4.4. Visualization of Results. Judging from the research results in Figure 6, the Portuguese team used more dribble breakthrough techniques in the game, and the success rate of dribble breakthroughs was also relatively high.

It is the excellent offensive players such as Ronaldo and Nani who can often create opportunities through sharp personal dribble breakthroughs. In fact, modern high-level football games require more comprehensive and threedimensional player skills. A team needs to have players with strong passing and control skills, as well as players with personal dribbling and breakthrough capabilities. This type of staffing will enrich the team's tactical play, making it more difficult for opponents to pin them.

4.5. Technical and Tactical Decision Based on Neural Network. Section 4.4 proved our algorithm to accurately predict the pass success rate of a single team. For opponents, the prediction of the time and fulcrum of the tackle (Figure 7 shows an example of tackle) will become extremely important.

This article uses neural network to make model prediction. Figure 8 below shows the predicted position and actual position. From Figure 8, we can intuitively see that the football technical and tactical command decision algorithm based on big data and neural network in this article has achieved effective results. It can provide a certain reference 


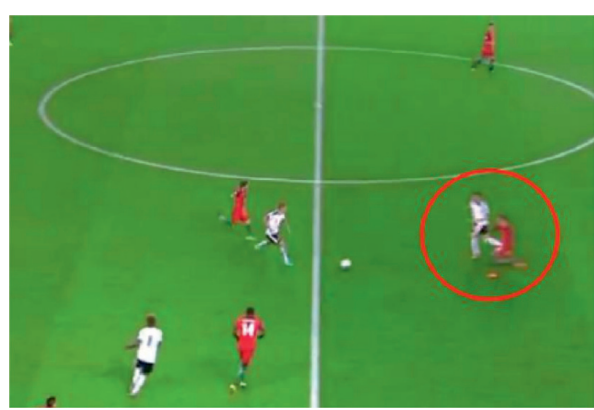

(a)

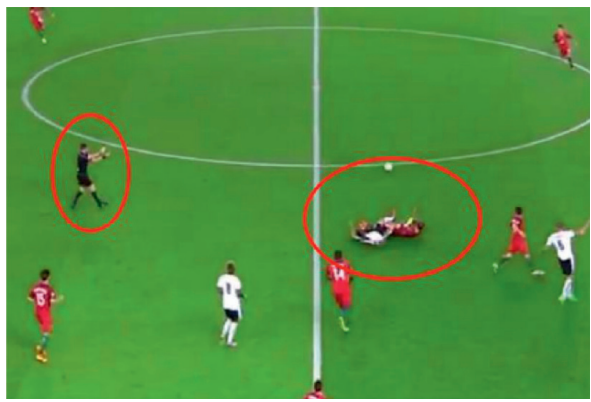

(c)

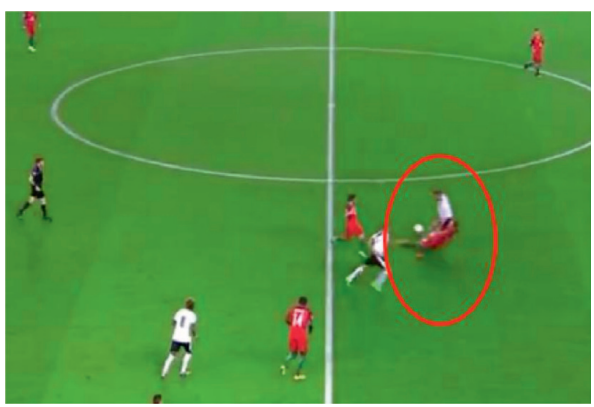

(b)

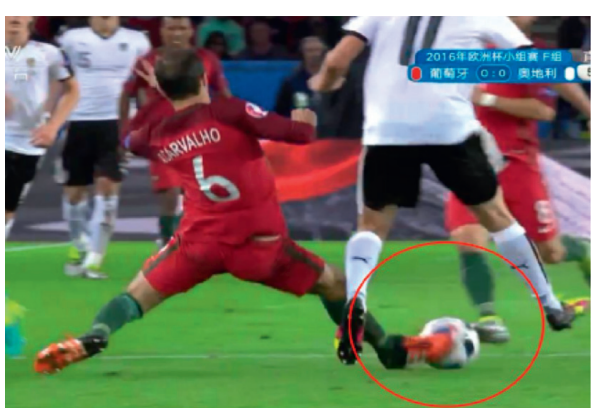

(d)

Figure 7: The neural network learns the time and location of the tackle. (a) Defensive position. (b) Tackle. (c) Referee's gesture. (d) Tackle slow motion replay.

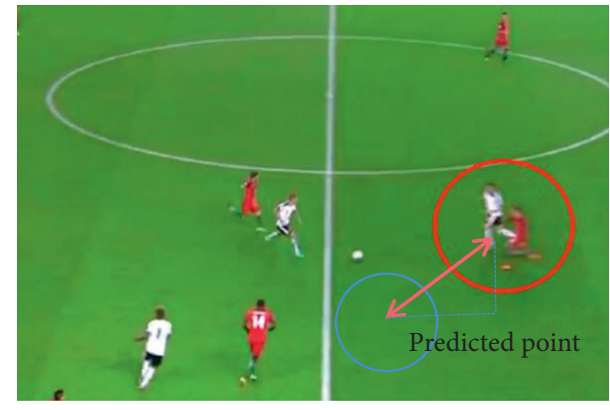

FIGURE 8: Predicted points and actual points.

value for the coaches and the training team's command and decision-making.

\section{Conclusion}

Based on big data and neural network technology, this paper designs a novel football technical and tactical command decision algorithm. Through, the experimental research proved that big data analysis can extract effective feature information. Secondly, we established a neural network model based on long- and short-term memory and proved through experiments that the algorithm can predict the pass success rate and defensive tackle position. It can formulate reasonable training plans for practitioners in the sports industry, especially coaches, and provide scientific command and decision-making advice on football standing skills. The method proposed in this paper has achieved good results in football technical and tactical command and decision-making and also provided some new ideas for the subject of football combined with computer technology.

\section{Data Availability}

The data used to support the findings of this study are included within the article.

\section{Conflicts of Interest}

The authors declare that there are no conflicts regarding the publication of this paper.

\section{Acknowledgments}

This work was supported by the Social Science Development Research Project of Hebei Province under Grant 20200502100 .

\section{References}

[1] D. Memmert, "Data analytics in football: positional data collection, modeling, and analysis," Journal of Sport Management, vol. 33, p. 574, 2019.

[2] H. Sarmento, A. Figueiredo, C. Lago-Peñas et al., "Influence of tactical and situational variables on offensive sequences during elite football matches," Journal of Strength and Conditioning Research, vol. 32, no. 8, pp. 2331-2339, 2018.

[3] F. Sors, M. Grassi, T. Agostini, and M. Murgia, "The sound of silence in association football: home advantage and referee bias decrease in matches played without spectators," European Journal of Sport Science, pp. 1-9, 2020.

[4] E. Elyakim, E. Morgulev, R. Lidor, Y. Meckel, M. Arnon, and D. Ben-Sira, "Comparative analysis of game parameters 
between Italian league and Israeli league football matches," International Journal of Performance Analysis in Sport, vol. 20, no. 2, pp. 165-179, 2020.

[5] R. Izzo, T. D’isanto, G. Raiola, A. Cejudo, N. Ponsano, and C. H. Varde'i, "The role of fatigue in football matches, performance model analysis and evaluation during quarters using live global positioning system technology at $50 \mathrm{~Hz}$," Sport Science, vol. 13, no. 1, pp. 30-35, 2020.

[6] M. J. Zammit, "Predictive analysis of football matches using in-play data," Master's thesis, University of Malta, Msida, Malta, 2018.

[7] X. Ning, K. Gong, W. Li, L. Zhang, X. Bai, and S. Tian, "Feature refinement and filter network for person re-identification," IEEE Transactions on Circuits and Systems for Video Technology, 2020.

[8] W. Cai and Z. Wei, "PiiGAN: generative adversarial networks for pluralistic image inpainting," IEEE Access, vol. 8, pp. 48451-48463, 2020.

[9] X. Ning, P. Duan, W. Li, and S. Zhang, "Real-time 3D face alignment using an encoder-decoder network with an efficient deconvolution layer," IEEE Signal Processing Letters, vol. 27, pp. 1944-1948, 2020.

[10] W. Cai and Z. Wei, "Remote sensing image classification based on a cross-attention mechanism and graph convolution," IEEE Geoscience and Remote Sensing Letters, 2020.

[11] X. Ning, K. Gong, W. Li, and L. Zhang, "JWSAA: joint weak saliency and attention aware for person re-identification," Neurocomputing, 2020.

[12] W. Cai, B. Liu, Z. Wei, M. Li, and J. Kan, “TARDB-Net: tripleattention guided residual dense and BiLSTM networks for hyperspectral image classification," Multimedia Tools and Applications, pp. 1-22, 2021.

[13] X. Ning, W. Li, B. Tang, and H. He, "BULDP: biomimetic uncorrelated locality discriminant projection for feature extraction in face recognition," IEEE Transactions on Image Processing, vol. 27, no. 5, pp. 2575-2586, 2018.

[14] Z. Wang, C. Zou, and W. Cai, "Small sample classification of hyperspectral remote sensing images based on sequential joint deeping learning model," IEEE Access, vol. 8, pp. 7135371363, 2020.

[15] W. Cai and Z. Wei, "Diversity-generated image inpainting with style extraction," 2019, http://arxiv.org/abs/1912.01834.

[16] Z. L. Yang, S. Y. Zhang, Y. T. Hu, Z. W. Hu, and Y. F. Huang, "VAE-Stega: linguistic steganography based on variational auto-encoder," IEEE Transactions on Information Forensics and Security, vol. 16, pp. 880-895, 2020.

[17] X. Ning, X. Wang, S. Xu et al., "A review of research on cotraining," Concurrency and Computation: Practice and Experience, 2021.

[18] D. Krzysztof and B. Pawet, "Analysis of goals and assists diversity in English premier league," Journal of Health Sciences, vol. 4, no. 5, pp. 47-56, 2014.

[19] M. Michalls, R. Joaquín González, A. Vasilis, and A. Rafael, "The creation of goal scoring opportunities in professional soccer," International Journal of Performance Analysis in Sport, vol. 19, no. 3, pp. 452-465, 2019.

[20] A Kubayi, "Analysis of goal scoring patterns in the 2018 FIFA world Cup," Journal of Human Kinetics, vol. 71, no. 1, pp. 201-210, 2019.

[21] J. Perl, A. Grunz, and D. Memmert, "Tactics analysis in soccer-an advanced approach," Dshs, vol. 12, 2013.

[22] T. Reilly and A. M. Williams, "International research in sports and exercise science including physiology, psychology, sports medicine and biomechanics, coaching and talent identification," Journal of Sports Sciences, vol. 23, no. 6, 2013.

[23] R. Pollard and C. Reep, "Measuring the effectiveness of playing strategies at soccer," Journal of the Royal Statistical Society: Series D (The Statistician), vol. 46, no. 4, pp. 541-550, 1997.

[24] L. R. Medsker and L. C. Jain, "Recurrent neural networks," Design and Applications, vol. 5, 2001.

[25] S. M. Arabzad, M. E. Tayebi Araghi, S. Sadi-Nezhad, and N. Ghofrani, "Football match results prediction using artificial neural networks; the case of Iran Pro League," Journal of Applied Research on Industrial Engineering, vol. 1, no. 3, pp. 159-179, 2014.

[26] F. Ameri, S. Moradian, T. M. Amani, and K. Faez, "The use of fundamental color stimulus to improve the performance of artificial neural network color match prediction systems," Jounal of Chemistry and Chemical Engineering, vol. 24, no. 36, 2005.

[27] C. D. M. Bezerra and C. J. Hawkyard, "Computer match prediction for fluorescent dyes by neural networks," Coloration Technology, vol. 116, no. 5-6, pp. 163-169, 2000.

[28] H. Li, "Analysis on the construction of sports match prediction model using neural network," Soft Computing, vol. 24, pp. 1-11, 2020.

[29] S. Booth, A. Shah, Y. Zhou, and J. Shah, "Sampling predictionmatching examples in neural networks: a probabilistic programming approach," 2020, http://arxiv.org/abs/2001.03076.

[30] Z. Wang, C. Long, G. Cong, and C. Ju, "Effective and efficient sports play retrieval with deep representation learning," in Proceedings of the 25th ACM SIGKDD International Conference on Knowledge Discovery \& Data Mining, pp. 499-509, Anchorage, AK, USA, July 2019.

[31] M. A. Russo, L. Kurnianggoro, and K. H. Jo, "Classification of sports videos with combination of deep learning models and transfer learning," in Proceedings of the 2019 International Conference on Electrical, Computer and Communication Engineering (ECCE), pp. 1-5, IEEE, Cox's Bazar, Bangladesh, February 2019.

[32] D. Tang, "Hybridized hierarchical deep convolutional neural network for sports rehabilitation exercises," IEEE Access, vol. 8, pp. 118969-118977, 2020.

[33] Y. C. Huang, I. N. Liao, C. H. Chen, T. U. İk, and W. C. Peng, "TrackNet: a deep learning network for tracking high-speed and tiny objects in sports applications," in Proceedings of the 2019 16th IEEE International Conference on Advanced Video and Signal Based Surveillance (AVSS), pp. 1-8, IEEE, Taipei, Taiwan, September 2019.

[34] J. Lee, S. Moon, D. W. Nam, J. Lee, A. R. Oh, and W. Yoo, “A study on sports player tracking based on video using deep learning," in Proceedings of the 2020 International Conference on Information and Communication Technology Convergence (ICTC), pp. 1161-1163, IEEE, Jeju Island, South Korea, October 2020 . 